\title{
Maria Theresia als „König von Ungarn“ im Krönungszeremoniell in Preßburg (1741)
}

\author{
Sandra Hertel (Wien)
}

Die Krönung Maria Theresias zum „König“ von Ungarn - zum „Rex Hungariae“ - am 25. Juni 1741 in Preßburg war ein politisch wie dynastisch bedeutsames Ereignis. ${ }^{1}$ Maria Theresia war seit Maria I. (1370-1395) die erste weibliche Thronfolgerin in Ungarn. Die Krönung im souveränen Königreich war ein wichtiger Schritt zur Festigung ihrer Herrschaft innerhalb der Habsburgermonarchie und zur Verbesserung der politischen Lage im Österreichischen Erbfolgekrieg. Sie markierte einen der Höhepunkte im „Schicksalsjahr“ 1741, in dem Maria Theresia ihre Herrschaft grundlegend festigte und die Legitimation ihrer Thronfolge definierte. Die Strategien ihrer Herrschaftsrepräsentation werden bei der Vorbereitung und Umsetzung des Krönungszeremoniells sowie den Öffentlichkeitsbestrebungen des Hofes sichtbar. Sie wurden durch die Notwendigkeit bestimmt, einerseits die dynastische Kontinuität ihrer Thronfolge durch die Tradition zu betonen und andererseits den durch ihr Geschlecht veränderten Umständen durch Anpassungen und Neuerungen gerecht zu werden. Die folgenden Ausführungen untersuchen das Zeremoniell und den Ablauf der ungarischen Krönung 1741 als Verfassungsfest ${ }^{2}$ und als Medienereignis. Maria Theresias Sonderrolle als weibliche Herrscherin und die damit einhergehenden Auswirkungen auf das Zeremoniell stehen dabei im Mittelpunkt der Betrachtung.

Die Krönung eines neuen Landesherrn war in der Frühen Neuzeit der festliche Höhepunkt seiner Investitur. Erbhuldigungen und Krönungen waren Verfassungsfeste, weil sie im Gegensatz zu höfischen und dynastischen Festen einen rechtsstaatlichen Charakter besaßen. Ausdruck dieser Verfassungsrelevanz waren die angewandten Rituale und Zeremonien des Festes, die auf althergebrachten Traditionen beruhten und sowohl die Anciennität als auch die Legitimität der Herrschaftswürde betonten. ${ }^{3}$ Diese performative Repräsentation diente der Sichtbarmachung komplexer Bedeutungszusammenhänge, die in der frühmodernen Ständegesellschaft nicht rational und diskursiv vermittelt werden konnten oder sollten. Symbole und Rituale ersetzten rationale Erklärungen und vielschichtige Bedeutungsebenen in der politischen Kommunikation. Die Repräsentation agierte sowohl als sinnstiftende als auch als sinnabbildende symbolische Handlung. ${ }^{4}$ Abstrahierte Phänomene wie Staat, Macht und Herrschaft bedurften der Vergegenwärtigung durch eine kollektiv verstandene Symbolsprache.

Frühneuzeitliche Krönungsfeste inszenierten und manifestierten gleichermaßen die ideale Gemeinschaftsordnung, die jeder Person einen festen Platz zuwies. Die Herrschererhebung war ein Staatsfest, an dem sämtliche soziale Gruppen einer Gesellschaft partizipierten - der Monarch, der höfische Adel, die städtischen Repräsentanten und die Bevölkerung. Rangunterschiede wurden durch das Festzeremoniell sichtbar gemacht, wodurch das Verfassungsfest die Ordnung herstellte, die es abzubilden beabsichtigte. ${ }^{5}$ Vor der Verschriftlichung von Verfassungen ersetzte das Fest mit seinen sinnlichen Eindrücken Rechtsnormen und politische Machtverhältnisse. Erst durch die sichtbare, öffentliche Durchführung der traditionellen Rituale legitimierte der Fürst seine Herrschaftsansprüche und nahm die Macht symbolisch und praktisch in Besitz. ${ }^{6}$ Daher war eine Krönung oder Erbhuldigung auch ein Übergangsritual, ${ }^{7}$ in dem der Fürst nicht nur eine neue Aufgabe annahm, sondern zur Verkörperung eines politischen Konzepts wurde. Im Krönungsritual wird diese Doppelnatur $^{8}$ des Königs deutlich: Zusätzlich zu seinem natürlichen Körper, dem body natural, nahm der König bei der Krönung den politischen Körper an, den body politic. ${ }^{9}$ Bei Maria Theresia ist die ,Zweikörperlichkeit' ihres Königtums aufgrund ihres Geschlechts noch offensichtlicher, denn ihr biologisch weiblicher Körper wurde durch die Salbung und Krönung zu 
einem politischen Staatskörper erweitert, der die vermeintlichen Schwächen der weiblichen Natur ausglich. ${ }^{10}$ Deshalb wurde Maria Theresia nicht zur Königin, sondern zum König von Ungarn gekrönt. Angesichts der 40 Jahre dauernden, bereits von Zeitgenossen dezidiert positiv beurteilten Regierung ${ }^{11}$ Maria Theresias ist die politisch schwierige, mitunter dramatische Anfangsphase ihrer Herrschaft im historischen Rückblick meist kaum präsent. Politisches Handeln und Repräsentation greifen im frühneuzeitlichen Staat ineinander und bedingen sich gegenseitig, daher sind Repräsentationsstrategien nicht ohne den politischen Kontext zu bewerten, der hier kurz skizziert werden soll.

\section{Die politische Ausgangslage 1741}

Ungarn war eine Ständevertretungsmonarchie, in der der König von den Ständen gewählt wurde; dieses Amt war nur Männern vorbehalten. Mit der Pragmatischen Sanktion, ${ }^{12}$ die auf dem Landtag 1722/23 angenommen wurde, bestätigten die Stände jedoch die untrennbare Verbindung ihres Landes mit dem Haus Habsburg, die erst in weiterer Konsequenz auch die weibliche Thronfolge einschloss. Mit dieser Änderung wurde das Recht der Stände auf freie Königswahl beschnitten und die formalrechtliche Selbstständigkeit der Monarchie zum Teil aufgehoben. ${ }^{13}$

Mit dem Tod Karls VI. am 20. Oktober 1740 trat die weibliche Erbfolge in den österreichischen Ländern ein. Bereits am selben Abend nahm Maria Theresia die Königstitel von Ungarn und Böhmen an und ließ sich von den obersten Hofbeamten huldigen, womit sie formalrechtlich das Herrschaftsamt übernahm. ${ }^{14}$ Am 22. November 1740 folgte die niederösterreichische Erbhuldigung, mit der sie auch symbolisch das Kernland der habsburgischen Monarchie in Besitz nahm. Diese Akte erfolgten wesentlich schneller als bei vorangegangenen Sukzessionen männlicher Thronfolger, weil sowohl der Hof als auch die Bevölkerung fest mit einem Krieg gegen Bayern rechnete, das die Pragmatische Sanktion nicht anerkannt hatte. ${ }^{15}$ Zudem äußerten sich aus der Bevölkerung Stimmen, die eine weibliche Landesherrin ablehnten und eine Zeit der Kriege und des politischen Chaos voraussahen. Eine weibliche Herrscherin war in Österreich ohne Vorbild und die Bedenken gegen
Maria Theresias Regierungsbefähigung waren weit verbreitet. ${ }^{16}$

Doch statt Bayern marschierte zuerst Preußens neuer König Friedrich II. im Dezember 1740 in Schlesien ein und löste so den Österreichischen Erbfolgekrieg aus. Für Maria Theresias Anerkennung seiner Usurpation bot er ihr seine Stimme für die Wahl Franz Stephans zum Kaiser an. Maria Theresia wollte jedoch keine Schwäche oder unehrenhafte Erpressbarkeit zeigen, sondern ihr Erbe militärisch verteidigen. Zur ersten Schlacht kam es am 10. April 1741, die der österreichische General Neipperg verlor. Der preußische Sieg führte zu einer Vereinigung der Feinde Österreichs im Nymphenburger Abkommen am 28. Mai 1741, in dem Spanien, Frankreich, Bayern, Sachsen und Preußen die Pragmatische Sanktion außer Kraft setzten und die österreichischen Länder unter sich aufteilten; Böhmen sollte an Bayern gehen und Mähren an Sachsen. ${ }^{17}$ Anfang Juni war die außenpolitische und militärische Lage von Maria Theresias Herrschaft somit denkbar schlecht.

Außer bei ihrer Hochzeit mit Franz Stephan von Lothringen 1736 war Maria Theresia bislang der Öffentlichkeit, also der Wiener Stadtbevölkerung, weitgehend verborgen geblieben. Bei bisher drei Geburten hatte Maria Theresia nur Töchter auf die Welt gebracht; Maria Elisabeth starb jedoch bereits im Sommer 1740, Maria Karolina folgte im Januar 1741. Das Ausbleiben eines männlichen Erben wurde als schlechtes Omen für die Regierung Maria Theresias gedeutet und die Rechtmäßigkeit ihrer Thronansprüche in Frage gestellt; entsprechend euphorisch und erleichtert wurde am 13. März 1741 die Geburt von Erzherzog Joseph begrüßt und gefeiert. Die Tatsache, dass Maria Theresia erst als Herrscherin einen Jungen zur Welt gebracht hatte, wurde nun als göttliche Segnung für ihre Thronansprüche interpretiert. ${ }^{18}$

Aufgrund ihrer Schwangerschaft konnte die wichtige Krönung in Ungarn nicht sofort zu Jahresbeginn 1741 durchgeführt werden. Traditionell ging der ungarischen Krönung der einmonatige Krönungslandtag der ungarischen Stände voran, der am 15. Mai 1741 eröffnet wurde. Am 27. Mai kamen 34 Deputierte der Stände zur Audienz bei Maria Theresia von Preßburg nach Wien und wurden von ihr in der Ratsstube ihres Appartements in der Hofburg empfangen. ${ }^{19}$

Erst Anfang Juni begannen die Vorbereitungen für die ungarische Krönung am 25. Juni im Preßburger 
1 Johann Daniel Herz: Der Zug Maria Theresias durch Preßburg. 1741, Albertina Wien, Inv.-Nr. DG48725.

Martinsdom. Maria Theresia berief eine Hofkonferenz ein, wie es vor wichtigen höfischen und dynastischen Ereignissen am Wiener Hof üblich war. In dieser Hofkonferenz berieten Hofbeamte und die wichtigsten Würdenträger des Hofes über das Festzeremoniell. ${ }^{20} \mathrm{Zu}$ ihren Aufgaben gehörte auch die Recherche in den höfischen Akten und Protokollen, um die jeweiligen Ereignisse nach historischen Vorbildern und Traditionen korrekt umzusetzen. Im Falle von Maria Theresias Krönung gab es bei der Wahl der Vorbilder Unstimmigkeiten: Obwohl klar war, dass Maria Theresia zum „König“ von Ungarn gekrönt werden sollte, diskutierten die Hofbeamten anhand einzelner Punkte, ob das Krönungszeremoniell ihres Vaters Karl VI. - als König von Ungarn Karl III. - von 1712 oder dasjenige ihrer Mutter Elisabeth Christine von 1714 als Vorbild dienen sollte. Doch um die Rechtslage korrekt abzubilden, musste
Maria Theresia unabhängig von ihrem Geschlecht zum König gekrönt werden. Würde sie wie eine Königin gekrönt werden, so gäbe es keinen ungarischen König, der das Land führen und Gesetze beschließen könnte. Eine Krönung ihres Ehemannes Franz Stephan von Lothringen zum ungarischen König war undenkbar, denn dieser war kein Habsburger. Das Gesetz verlangte einen König und dieser konnte laut der Pragmatischen Sanktion nur Maria Theresia sein. In der praktischen Umsetzung sollte der Tatsache, dass Maria Theresia eine Frau war, entsprechend wenig Gewicht beigemessen und die Thronfolge als so reibungslos wie möglich dargestellt werden. Das entsprach auch den Intentionen von Maria Theresia selbst. Neben den Nachfragen der Hofkonferenz zum Krönungszeremoniell im Protokoll notierte sie lapidar: „es ist nichts neues, also sich nach dem vorigen Mahl zu richten. ${ }^{\text {“21 }}$ 
2 Skizze des Martinsdoms für den Krönungsgottesdienst (Ausschnitt). Haus-, Hof- und Staatsarchiv, Ältere Zeremonialakten 40, Konv. 11, Fol. 190r. Legende: Nr. 1: Altar. Nr. 2: Ordinari-Thron. Nr. 5: Inthronisations-Thron. Nr. 8: Gerüst für die Erzherzoginnen Maria Anna und Maria Magdalena sowie Franz Stephan. Nr. 16: Sitzbänke für die Hofdamen.

\section{Die Elemente des ungarischen Krönungszeremoniells}

Das ungarische Krönungszeremoniell bestand aus fünf Elementen, die in allen Königskrönungen seit Maximilian II. im Jahre 1563 Anwendung fanden: ${ }^{22}$ Ein- und Umzug des Herrschers, Krönung, Standeserhebung, Verfassungseid und Krönungsritt mit Schwertstreichen. Damit enthielt das ungarische Krönungszeremoniell alle relevanten Elemente, die das frühneuzeitliche Verfassungsfest auszeichneten und somit auch bei anderen Krönungsfesten, etwa bei der Kaiserkrönung, zu finden sind. ${ }^{23}$ Auf diese Elemente soll im Folgenden detailliert eingegangen werden.

Am 20. Juni 1741 zog Maria Theresia aus dem österreichischen Wolfsthal kommend in die ungarische Krönungsstadt Preßburg ein. Zunächst trafen sich städtische und ständische Repräsentanten mit Maria Theresia in einem mit türkischen Teppichen geschmückten Zelt vor der Stadt. Anschließend folgte der feierliche Einzug Maria Theresias in Preßburg. Vor dem Wiener Tor wurde ihr der Stadtschlüssel überreicht, um symbolisch die Unterwerfung der Stadt unter die Macht des Herrschers anzuzeigen. ${ }^{24}$ Der feierliche Herrschereinzug - Adventus - ist ein antikes Festritual, das im 16. Jahrhundert in Europa wieder verstärkt im Herrschaftszeremoniell genutzt wurde. ${ }^{25} \mathrm{Im}$ festlichen Einzug präsentierte sich der Monarch mit seiner prachtvollen Entourage aus Dienern, Pferden und kostbar geschmückten Wagen der Öffentlichkeit. ${ }^{26}$ Die Bevölkerung blieb bei diesem Festakt nicht passiv, sondern war durch ihre bloße Anwesenheit, die Akklamationsrufe "Vivat" und ihre emotionalen Äußerungen des Erstaunens, der Begeisterung oder der Freude ein wesentlicher 
Bestandteil der Gesamtinszenierung des Herrschereinzugs. ${ }^{27}$

Wichtiger noch als der Einzug der künftigen Königin Maria Theresia in Preßburg am 20. Juni 1741 waren ihr Krönungszug zum Martinsdom am 25. Juni 1741 sowie der anschließende Zug durch die Stadt. Die Prozession wurde von Glockengeläut, Kanonenschüssen, Vivat-Rufen sowie Pauken und Trompeten akustisch begleitet. Maria Theresia saß in einer offenen Kutsche in einem eigens für dieses Ereignis angefertigten Krönungskleid: „Ihro allerhöchst gedacht königl. May. sassen alleine in ged. chaisen und waren in hungarischer Kleydung, wovon der Rock von weissen Crissette durch aus mit gold gesticket, und sehr reich en dessein mit Rubin Schmaragden, und Brillanten besetzet. ${ }^{\text {}} 28$

Ein bedeutsames Element des Herrscherumzugs war der Auswurf von Münzen. Im ungarischen Krönungszeremoniell übernahm der ungarische Kammerpräsident traditionell diese Aufgabe; 1741 erfüllte Georg Graf Erdödy diese Funktion..$^{29}$ Durch den Auswurf von Gedächtnismünzen demonstrierte Maria Theresia ihre Clementia - die Freigebigkeit, eine der wichtigsten Herrschertugenden seit dem Mittelalter. ${ }^{30}$ In der Habsburgerdynastie spielte die Clementia eine über die allgemeine Bedeutung hinausgehende Rolle als die zur erblich bedingten Familieneigenschaft erklärte Clementia austriaca ${ }^{31}$ Maria Theresia übernahm diese habsburgische Tugendtradition und erklärte nach der Justitia die Clementia zu ihrem Regierungsmotto, das auch auf den ausgeworfenen Krönungsmünzen zu lesen war. Die mitunter gewalttätigen Auseinandersetzungen um die Gaben des Herrschers, zu denen auch Wein und gebratenes Ochsenfleisch zählten, gehörten ebenso zu den Merkmalen eines Verfassungsfests und stellten als affektgeleitete Handlungen einen wirkungsvollen Kontrast zu der geordneten zeremoniellen Inszenierung des Herrscherumzugs dar. ${ }^{32}$ Zudem war es Tradition, die Aufbauten der öffentlichen Plätze und auch den mit Brettern und Tüchern ausgelegten Fußweg, nach den Feierlichkeiten der Plünderung durch das Volk zu überlassen. ${ }^{33}$

Die Krönung und Salbung der Königin fand im Martinsdom im Rahmen eines Gottesdienstes statt. Erst durch das Aufsetzen der apostolischen Stephanskrone erhielt Maria Theresia die Segnung Gottes und die offizielle Anerkennung aller Ungarn als ihre Königin.
Dieses Ritual war der Mittelpunkt des Verfassungsfests und vereinigte die religiösen, rechtlichen und symbolischen Elemente der Krönung.

\section{Vorbereitungen und Ablauf von Maria Theresias Krönung}

Bei den Vorbereitungen spielte die Ausgestaltung des Martinsdoms eine große Rolle. Dazu gehörten Dienstanweisungen für die Hoftapezierer, die den Martinsdom mit persischen Teppichen, Gobelins und einem Baldachin schmückten, Sitzordnungen für die Messe und ein vollständiges Programm des Ablaufs der Festlichkeiten. ${ }^{34}$ Eine Änderung der üblichen Sitzordnung musste erfolgen, weil zum ersten Mal bei der Krönung eines ungarischen Königs im Chorraum exponierte Sitzgelegenheiten für Hofdamen geschaffen werden mussten. ${ }^{35}$ Aus Gründen der Sittlichkeit durfte kein Mann Maria Theresia bei den Umzügen vor und nach der Salbung assistieren. Die äußerst prominente Positionierung der Hofdamen während eines ungarischen Krönungsgottesdienstes stellte eine einzigartige Ausnahme dar, die allein durch Maria Theresias Geschlecht möglich und notwendig war. An Höfen, an denen eine Fürstin und nicht ein Fürst den höchsten Rang bekleidete, hatten auch die sie umgebenden und bedienenden Hofdamen einen höheren Rang als männliche Adelige des Hofes. $^{36}$

Der Krönungsgottesdienst entsprach einer ,theatralen' Inszenierung mit einer festlich hergerichteten Bühne sowie einer Handlungschoreografie, wie eine Skizze des Kircheninneren deutlich macht. In der Mitte des Altarraums stand der "Ordinari-Thron“ unter einem Baldachin, von dem aus Maria Theresia den Beginn des Gottesdienstes verfolgte. Nach der Salbung und Krönung, die vor dem Altar stattfand, wurde sie auf dem höher stehenden Thron auf der Evangelienseite inthronisiert. Maria Theresias wichtigste Wege waren eingezeichnet, um einen reibungslosen Ablauf der Zeremonien durch entsprechende Information aller Beteiligten zu ermöglichen. Wichtig waren ihr Gang von der Sakristei zum Thron und der Auszug aus dem Seitenausgang nach der Inthronisation. Vor der Salbung ging Maria Theresia hinter den Altar, um sich von ihren Hofdamen Brust, Schulter und Hände für die Salbung freilegen 
zu lassen; das Öl wurde nach der Salbung auch dort wieder durch die Hofdamen mit Tüchern entfernt. Die inkognito angereisten Familienmitglieder, Franz Stephan und die Erzherzoginnen Maria Magdalena und Maria Anna, sahen dem Krönungsakt von einer Tribüne im Kirchenfenster zu.

Franz Stephan stand als Lothringer und Großherzog der Toskana im Rang hinter seiner Schwägerin Maria Anna und der Tante seiner Ehefrau, Erzherzogin Maria Magdalena. Beim Krönungsbankett saßen die beiden Erzherzoginnen neben Maria Theresia, während Franz Stephan erst nach Maria Anna einen Platz an der Tafel erhielt. ${ }^{37}$ Dadurch stellte Maria Theresia die Habsburgerdynastie als legitime Landesherren und ihre eigene Herrschaft in den Vordergrund; ihre eheliche Verbindung hatte für die Königskrönung keine Bedeutung. Erst auf dem Landtag im September 1741 ließ sie Franz Stephan zum Mitregenten ernennen.

Obwohl Maria Theresia und ihr Obersthofmeister Rudolf Graf Sinzendorf deutlich gemacht hatten, dass das Krönungszeremoniell dem Vorbild von Karl III. (VI.) folgen sollte, traten zahlreiche Nachfragen innerhalb der Hofkonferenz auf. So wurde vorgeschlagen, dass Maria Theresia mit einer Hauskrone auf dem Kopf zur Krönung gehen sollte, wie dies bei der Krönung von Maria Theresias Mutter Elisabeth Christine 1714 der Fall gewesen war. Diesem Vorschlag widersprach der Obersthofmeister mit dem Argument, dass dies bei den Krönungen von Joseph I. und Karl III. nicht der Fall gewesen sei und „Eure königliche Majestät nicht als eine gemahlin eines Königs, sondern wie ein König selbst gecrönt werden. “38 Der wesentliche Unterschied in den Krönungszeremonien bestand darin, dass ungarische Königinnen nicht mit der Stephanskrone gekrönt wurden; diese wurde ihnen während der Salbung lediglich über die rechte Schulter gehalten. ${ }^{39}$ Schließlich einigte sich die Hofkonferenz auf den Vorschlag, Maria Theresia solle auf dem Weg zum Martinsdom „einen Aufputz in form einer Cron aufhaben“" ${ }^{40}$ der vor der Krönung leicht abzunehmen wäre. Maria Theresia kommentierte diesen Vorschlag lapidar mit: „das können wohl die Männer sagen, die nichts von unserem aufputz verstehen ${ }^{\text {"41 }}$ und fuhr letztlich ohne Kopfschmuck zur Krönungsfeier.

Die theoretische Trennung von männlichem body politic und weiblichem body natural sorgte für
Unbehagen und Verwirrung. Dass diese politische Konstruktion den gesellschaftlichen Normen nicht entsprach und sogar innerhalb des Hofes für Unverständnis sorgte, ist ein weiterer Beleg für die komplexen Schwierigkeiten, die mit Maria Theresias Regierungsantritt einhergingen. Umso wichtiger war eine eindeutige und nicht hinterfragbare symbolische Inszenierung in der Öffentlichkeit, die alle Unsicherheit von vornherein ausschloss und eine eindeutige Interpretation nahelegte.

Ein weiterer Verhandlungspunkt, bei dem Maria Theresias biologisches Geschlecht dem männlichen Krönungsritus im Weg zu stehen schien, war die Handhabung des Krönungsschwerts. Der ungarische Hofrat Koller sprach sich dagegen aus, dass Maria Theresia während der Krönung die drei traditionellen Schwertstreiche mit dem Stephansschwert durchführte und dieses auch während der weiteren Zeremonien umgegürtet trug. Wieder führte er das Argument an, dass ungarische Königinnen dieses Ritual während ihrer Krönungen nicht ausführten. Hier ist aber nicht nur das traditionelle Zeremoniell, sondern auch die Objektsymbolik des Schwerts von Bedeutung. Das Schwert war seit der Antike ein weit verbreitetes Symbol für Männlichkeit und Stärke. ${ }^{42}$ Es stand für die militärische wie körperliche Potenz und Stärke eines Herrschers und somit für Gewalt und Aggressivität, die dem weiblichen Tugendkanon diametral gegenüberstanden. Die künstlerische Darstellung einer Frau mit Schwert beschränkte sich in der Frühen Neuzeit weitgehend auf die abstrakte Personifikation der Justitia. ${ }^{43}$ Auf Porträts realer Frauen wurde das Schwert hingegen nie verwendet, weil es dem männlichen Handlungsspielraum zugeordnet war. Aus diesem Grund sprachen sich wohl einige Hofbeamte gegen den Gebrauch des Schwerts durch Maria Theresia aus. Doch das Schwert symbolisierte auch den Schutz eines Landes durch den Herrscher sowie dessen weise und rechtmäßige Herrschaft. Gerade deshalb spielte das Schwert für die Versinnbildlichung der Herrschaft Maria Theresias in Ungarn eine zentrale Rolle in der repräsentativen Inszenierung des Krönungszeremoniells.

Eine kleine Abänderung in der Handhabung dieser wichtigen Krönungsinsignie gab es dennoch. Die Hofkonferenz befürchtete, dass das Tragen des schweren Schwerts eine zu große Last für Maria Theresia darstellen könnte. Deshalb wurde das 
Schwert außerhalb seines zeremoniellen Gebrauchs von dem zuständigen Magister auf einem samtenen Polster getragen. Diese Vorkehrung geschah im Bewusstsein der Medienwirksamkeit des Krönungsfestes. Das ganze Zeremoniell war auf die Betonung und Sichtbarmachung der Stärke und Legitimität der Herrscherin zugeschnitten. Ein aufgrund der körperlichen Schwäche der Königin zu Boden fallendes Schwert hätte eine katastrophale symbolische Wirkung gehabt. Aus demselben Grund zog Maria Theresia bei der Krönung auch nicht die Sandalen des heiligen Stephan an; sie konnte in diesen nicht laufen, ohne umzuknicken. Die Interpretation des Stolperns als Unfähigkeit der Königin war in einer Gesellschaft, die jedes Ereignis als Wink des Schicksals deutete, unbedingt zu vermeiden. ${ }^{44}$

Doch auch diese Tradition wurde im Sinne der Königskrönung beibehalten. Maria Theresia führte mit dem Schwert die traditionellen drei Kreuzstreiche in der Kirche aus, während die Gemeinde „Vivat Domina, Rex noster" rief. ${ }^{45}$ Anschließend wurde Maria Theresia vom Erzbischof von Gran gemeinsam mit dem Palatin Johann Graf Pálffy die Stephanskrone auf das Haupt gesetzt. In vollem Ornat, mit Zepter und Reichsapfel in den Händen, nahm Maria Theresia nun auf dem höheren Inthronisations-Thron Platz, womit die Krönung offiziell vollzogen und sie als König von Ungarn eingesetzt und bestätigt war. Erneut rief die Gemeinde „Vivat Domina, Rex noster". Diesmal war der Akklamationsruf so laut, dass er auch nach außen drang und von der Bevölkerung vor der Kirche aufgegriffen und durch weitere laute Rufe durch die ganze Stadt weitergetragen wurde. ${ }^{46}$ Durch dieses Ritual partizipierte das Volk ebenfalls an dem im geschlossenen Sakralraum stattfindenden Krönungszeremoniell. Der Akklamationsruf - zu Deutsch: „Es lebe die Herrin, unser König“ - betonte erneut die Doppelnatur der Königin, die zwar das weibliche Geschlecht, aber auch ein männliches Amt verkörperte. Sie war Herrin und König und symbolisierte trotz ihres dynastisch weniger wertvollen Geschlechts die Kontinuität und Würde des unsterblichen Staatskörpers. In dieser Verkörperung war sie rechtmäßiger Herrscher, und diese Botschaft richtete sich insbesondere an ihre Gegner im Österreichischen Erbfolgekrieg.

Nach der Salbung ging Maria Theresia zu Fuß durch die Straßen Preßburgs zur Franziskanerkirche, wo sie
48 Personen zu Rittern vom Goldenen Sporn schlug. Dieses Ritual betonte den korporativen Aspekt der Krönung als einen Vertrag zwischen dem König und den ungarischen Magnaten. Als neuer Herrscher begann Maria Theresia ihre Regierung mit der Auszeichnung neuer Würdenträger, die ihr während ihrer Regentschaft dienen sollten. Ein Herrschaftswechsel führte auch zu neuen Dienstverhältnissen, wodurch der Dienst nicht an den Staat, sondern an das Leben des Königs gebunden war. Die Standeserhebung war Bestandteil mehrerer europäischer Krönungsrituale, darunter auch der Kaiserkrönung in Frankfurt. ${ }^{47}$

Das vierte relevante Element des ungarischen Krönungsfestes war der Schwur des neuen Herrschers auf die ungarische Verfassung. Der öffentliche Eid vor dem Kloster der Barmherzigen Brüder wurde von allen Königen praktiziert und war keinesfalls ein rein ritueller Akt, sondern ein bedeutsamer staatsrechtlicher Aspekt des Krönungsfests. ${ }^{48}$ Der auf einem öffentlichen Platz unter freiem Himmel abgelegte Schwur war ein Vertrag zwischen Königin und Untertanen, der ihre Pflichten in den Mittelpunkt stellte. Die zuvor und anschließend getätigten Akklamationsrufe der anwesenden Bevölkerung bestätigten stellvertretend für alle Ungarn deren Anteil an dem Vertrag mit der Königin. Somit kam dieser repräsentative Akt einem unmittelbaren Vertragsabschluss mit dem Volk gleich. ${ }^{49}$

Als letztes Krönungsritual absolvierte Maria Theresia den Ritt auf dem Krönungshügel am Donauufer, bei dem sie mit dem Krönungsschwert die symbolischen vier Streiche in alle Himmelsrichtungen ausführte. Dieser Aspekt des ungarischen Krönungszeremoniells ist im Vergleich $\mathrm{zu}$ anderen europäischen Krönungszeremonien einzigartig. Der Einzug des Fürsten zu Pferd als Krönungsritt war Element mehrerer frühneuzeitlicher Inaugurationen, doch die gleichzeitig ausgeführten Schwertstreiche verliehen dem Ritual eine besonders symbolträchtige Komponente. Als ungarisches Spezifikum genoss er in der Bevölkerung größte Beliebtheit, war für die Herrscher aber auch riskant. Nach der Abschaffung der Ritterturniere als Bestandteil von Krönungen im 16. Jahrhundert ist der ungarische Krönungsritt das letzte Herrscherritual, das die Sportlichkeit und Geschicklichkeit des Monarchen erforderte - Eigenschaften, die im 18. Jahrhundert eigentlich nicht mehr zum Tugendkanon eines Königs gehörten. 
Im Gegensatz zu ihrem Vater Karl absolvierte Maria Theresia den Zug durch die Stadt am Krönungstag nicht zu Pferd, sondern in einer offenen Kutsche. ${ }^{50}$ Maria Theresia konnte mit ihrem prachtvollen Krönungskleid in einer breiten , chaise ' weit repräsentativer zur Schau gestellt werden und sich auch sicherer durch die Stadt bewegen als hoch zu Ross. Der Ritt auf den Krönungshügel sollte jedoch nicht umgangen werden: „hierauff erhuben sich allerhöchst gedacht Ihro May. zu Pferde, und also ritten in vollem Galop den Königsberg allein hinauf, zogen daselbst das Schwerd des heyligen Stephani aus, und thaten damit gegen die 4 Theile der Welt in form eines Creutzes 4 streiche $[\ldots]$ sich jeder Zeit mit dem Pferde wendende, anzuzeigen, das Sie solches Königreich von allen Theilen vor denen feinden beschützen wolle, und ritten darauf in vollem Galop wieder herunter unter beständigen Vivat zuruffen des unzahlbar da herum versamlet gewesenen Volcks [...]. ${ }^{{ }^{51}}$

\section{Maria Theresias Krönung als Medienereignis}

Die ungarische Krönung Maria Theresias im Jahre 1741 war ein Medienereignis, ${ }^{52}$ das vom Hof bewusst in die Öffentlichkeit getragen und vom interessierten Zeitungspublikum in ganz Europa wahrgenommen wurde. Öffentlichkeit in der Frühen Neuzeit ist ein vieldiskutierter und mittlerweile auch gut erforschter Begriff. Allgemein spricht man von mehreren Öffentlichkeiten: der höfischen Öffentlichkeit, die den Diskursraum der europäischen Höfe und sein Nachrichtennetz definiert und auf die vielfache gegenseitige Beobachtung der im Prestigewettkampf stehenden Höfe verweist ${ }^{53}$ sowie geographisch wie sozial begrenzte Teilöffentlichkeiten außerhalb der Höfe, die in unterschiedlicher Weise an der Nachrichtenverbreitung partizipierten. ${ }^{54}$ Die mündliche Weitergabe von Nachrichten dominierte auch noch im 18. Jahrhundert, weil sie im Vergleich zur Verbreitung in gedruckten Zeitungen und Flugschriften wesentlich schneller und einfacher erfolgte. Daher ist auch die Partizipation der niederen Bevölkerungsschichten am Nachrichtenmarkt und an den Meinungsdiskursen nicht zu unterschätzen. ${ }^{55}$

Über Zeitungen, politische Druckschriften und Bücher nahm die bürgerliche und gebildete
Öffentlichkeit Informationen des Hofes und der Eliten auf. Ihre Nachfrage nach bestimmten Themen und Autoren steuerte die Produktion und war eine direkte Resonanz auf die publizistischen Erzeugnisse. Dadurch standen diese Öffentlichkeiten auch in einem sich wechselseitig beeinflussenden Kommunikationsverhältnis mit der Obrigkeit. ${ }^{56}$

Die höfischen Strategien der Öffentlichkeit lassen sich in vier Phasen unterteilen: Ankündigung, detaillierte Berichterstattung, Einbeziehung des Publikums und Verewigung des Ereignisses im kollektiven Gedächtnis. ${ }^{57}$ Sie traten bei herrschaftsrelevanten Momenten in Erscheinung, die zu gemeinsamen Ereignissen von Herrscher und Untertanen stilisiert und medial verstärkt wurden. Diese vier Phasen sind auch bei der ungarischen Krönung Maria Theresias zu beobachten.

Die Ankündigung des Ereignisses erfolgte durch das Wienerische Diarium, eine mit kaiserlichem Privileg des Hofes herausgegebene, zweimal wöchentlich erscheinende deutschsprachige Zeitung. Das Wienerische Diarium veröffentlichte Postberichte aus dem europäischen Ausland und berichtete über Ereignisse in Wien, die sich in erster Linie auf die Aktivitäten der kaiserlichen Familie und des Hofes beschränkten..$^{58}$ Aufgrund der Nähe zum Wiener Hof besaß das Diarium eine große Relevanz und wurde auch in anderen erbländischen Städten wie etwa im nahen Preßburg gelesen. Das Wienerische Diarium berichtete, wie viele andere Lokalzeitungen in Residenzstädten, hauptsächlich über höfische Ereignisse, die nicht nur in ihrem Ablauf beschrieben, sondern bisweilen auch hinsichtlich des Zeremoniells erläutert wurden. ${ }^{59}$ Zwischen dem Herausgeber des Wienerischen Diariums, dem Hofbuchdrucker Johann Peter van Ghelen, und dem Obersthofmeisteramt bestand ein reger Informationsaustausch, was sich nicht zuletzt auch in den ähnlichen Formulierungen in den Zeremonialprotokollen des Hofes und im Wienerischen Diarium manifestierte. ${ }^{60}$

Die Ankündigung der bevorstehenden Krönung Maria Theresias weckte das Interesse der Bevölkerung an dem Ereignis. Die begleitende Berichterstattung lieferte Erklärungen und Hintergrundinformationen zum sinnlich-ephemeren Festerleben und vermittelte die Außergewöhnlichkeit des Moments. ${ }^{61}$ Bereits drei Wochen nach der erfolgten Krönung wurde im Wienerischen Diarium die ausführliche Beschreibung 
der Feierlichkeiten ${ }^{62}$ als Druckschrift für 17 Kreuzer angeboten. ${ }^{63}$ Sie wurde nach Genehmigung des Hofes durch den Hofbuchdrucker van Ghelen verlegt und erschien in identischer Form auch in anderen Verlagen im Reich. ${ }^{64}$ Die Erläuterungen des Zeremoniells verwiesen auf die historische Tradition und repetierten identitätsstiftende Fakten und Mythen, die sich im kollektiven Gedächtnis der Leserschaft festigen sollten. $\mathrm{Zu}$ den sinnstiftenden Elementen der ungarischen Krönung zählten auch die Insignien selbst. In der Krönungsbeschreibung wird behauptet, dass kaum eine andere Nation die königliche Krone mit mehr Respekt verehre. ${ }^{65}$ Die historisch-sakrale Herkunft der Krone, die die Vereinigung des weltlichen und des himmlischen Königreichs symbolisieren sollte, wird in einem Exkurs beschrieben, wodurch der Leserschaft die Außergewöhnlichkeit des ungarischen Königtums und der Krönungszeremonie vor Augen geführt wurde. Dadurch evozierte man bewusst den mystisch-emotionalen Aspekt des Ereignisses als erwünschte Reaktion beim Lesepublikum. Die enge Verbindung der Bevölkerung zur ungarischen Krone diente der Herrscherrepräsentation Maria Theresias und fand als legitimitätsstiftende Handlung ihren Platz im Krönungszeremoniell. Die Krone und die Krönungskleinodien wurden nicht nur am Abend vor der Krönung in einem eigenen, sehr festlichen Umzug durch die Stadt in den Martinsdom gebracht, sondern auch an den drei Tagen nach der Krönung im Turm des Preßburger Schlosses zur öffentlichen Besichtigung ausgestellt. ${ }^{66}$

Dieser Aspekt beleuchtet zugleich die nächste Phase der Öffentlichkeitsstrategie - die Einbeziehung der Bevölkerung in das Verfassungsfest. Als Zuschauer der Einzüge und rituellen Handlungen auf den Plätzen und Straßen der Stadt war die Bevölkerung nicht nur ein passiver Adressat von herrschaftlicher Prachtentfaltung, sondern auch Teilnehmer an der gemeinschaftlichen Repräsentation des Königtums. Ihre Anwesenheit und die Akklamation des Geschehens durch die Vivat-Rufe legitimierten das Verfassungsfest und ermöglichten erst dessen Rechtsgültigkeit. ${ }^{67}$

Für eine nachhaltige Verankerung des ephemeren Erlebnisses sorgten, neben detaillierten Beschreibungen, auch weitere Medien wie Predigten, Stiche oder Medaillen. Gerade Letztere waren eine beliebte Kunstgattung der Herrschaftsrepräsentation, weil sie
Botschaften auf besonders kleinem Raum verdichteten. ${ }^{68}$ Auch die Krönungsmünzen, so genannte Auswurfmünzen oder Gedenkpfennige, ${ }^{69}$ die anlässlich von Maria Theresias Krönung vom Hauptmünzamt geprägt und unter das Volk verteilt wurden, sollten die Beschenkten an das persönliche Erleben erinnern. Auf dem Avers der Münze schwebte die Stephanskrone über der Inschrift: „MARIA / THERESIA / IN REG · HUNGARIAE / CORONATA / POSON · 25 - IUN / 1741“70 Das Revers zeigte den böhmischen Löwen mit dem ungarischen Patriarchenkreuz und dem österreichischen Bindenschild, wodurch die drei Herrschaftswürden Maria Theresias ikonographisch miteinander verschmolzen; darüber prangte ihr Regierungsmotto "Justitia et Clementia“ als weitere Inschrift. ${ }^{71}$ Auch ohne umfassende Lateinkenntnisse konnte die einfache Bevölkerung, die mit diesen Gedächtnismünzen beschenkt wurde, die Herrschaftszeichen und das Regierungsmotto deuten. Die komplexeren emblematischen und allegorischen Bedeutungen und Sinnzusammenhänge mögen ihnen verschlossen geblieben sein, doch wurde gerade dadurch die überhöhte Würde des Königtums deutlich, das sich als Mysterium präsentierte - eine umfangreiche Belehrung der Bevölkerung in herrschaftlicher Ikonographie wurde nicht angestrebt. ${ }^{72}$ Für interessierte Leser bot die Krönungsbeschreibung jedoch eine Erklärung der Darstellungen und Inschriften auf den Auswurfmünzen. ${ }^{73}$ Die anlässlich der Krönung vom Hauptmünzamt geschlagenen Medaillen hingegen richteten sich an ein elitäreres Publikum, das mit der Symbolsprache der Medaillen vertraut war und die darin transportierten Botschaften zu deuten wusste; zu diesen gehörten auch wichtige ungarische Würdenträger, die die Medaillen als Auszeichnung erhielten.

In den Erblanden spielte die Krönung in Ungarn eine große Rolle, weil sie auch die Festigung der Herrschaft im österreichischen Kernland beförderte. In Wien wurde am 29. Juni 1741 ein feierlicher Dankgottesdienst im Dom von St. Stephan abgehalten; die Predigt des Jesuiten Franz Peikhardt wurde in der Folge auch als Druckschrift publiziert. ${ }^{74}$ Auch in der vorderösterreichischen Stadt Freiburg im Breisgau wurde ein Dankfest gefeiert, das als Loyalitätsbekundung gegenüber der habsburgischen Landesherrin $\mathrm{zu}$ verstehen ist; ${ }^{75}$ Freiburg war in der Vergangenheit mehrfach französischen Angriffen ausgesetzt 
gewesen und befand sich zu Beginn des Österreichischen Erbfolgekriegs durch den französischen Kriegsbeitritt wieder in einer gefährlichen Lage.

Die gedruckten Stiche und Beschreibungen richteten sich nicht nur an die politisch interessierten Beobachter innerhalb der Erblande, sondern auch im Ausland. So war die ungarische Krönung auch Thema in der halbjährlich in Frankfurt erscheinenden Zeitschrift Relationis Historicae [...] Continuatio, in der ein Stich die Stephanskrone und das Innere des Martinsdoms während der Krönung zeigte. ${ }^{76}$ Die Zeitschrift war ein Nachrichtenmedium für das Heilige Römische Reich und fasste wichtige politische Ereignisse der vorangegangenen Monate zusammen. Dennoch sind von der ungarischen Krönung vergleichsweise wenige Berichte oder Stiche erhalten. Das lag wahrscheinlich an der zeitlichen Nähe zu einem anderen wichtigen Medienereignis, der Geburt des Thronfolgers Joseph am 13. März 1741, das mit einem großen Volksfest und einer prachtvollen Illumination der Stadt Wien durch Adelige und Bürger gefeiert wurde. Die Sicherung der Thronfolge war das Großereignis des Frühjahrs, das zu einer umfangreichen Produktion von Druckschriften führte, die im Juni zum Verkauf angeboten wurden. Das Bedürfnis der Leserschaft nach weiteren Huldigungen an die neue Landesherrin war damit offenbar bereits gedeckt, sodass weitere Privatinitiativen von Autoren und Verlegern wohl auch aus wirtschaftlichen Gründen unterblieben. Druckgrafiken der Krönungsfeierlichkeiten waren hingegen beliebter; allein im Wienerischen Diarium wurden drei Stiche zum Kauf angeboten, ${ }^{77}$ die in Wien beim Hofbuchdrucker van Ghelen und in Preßburg bei Michael Kochberger $\mathrm{zu}$ erwerben waren. Die drei Bildmotive waren der Zug Maria Theresias durch die Stadt, die Krönung im Martinsdom und der Ritt auf dem Krönungshügel.

Das Element des Krönungsritts spielte in der Nachwirkung des Medienereignisses eine besondere Rolle. Dessen sichere Ausführung und die geschickte Handhabung des Schwertes waren eine wichtige Botschaft für alle Zweifler an Maria Theresias Herrschaftsbefähigung im In- und Ausland; deshalb wurde dieser Akt in allen Krönungsbeschreibungen deutlich hervorgehoben. ${ }^{78}$ Er wurde auch auf mehreren Krönungsmedaillen verewigt und war das einzige ephemere Festelement der ungarischen Krönung, das auf eine Medaille geprägt wurde. ${ }^{79}$ Das Motiv war nicht nur
3 Matthäus Donner: Krönung zur Königin in Ungarn. KHM Wien, Münzkabinett, Inv.-Nr. 1.885bß.

ein spezifisch ungarisches Element, sondern besaß zudem einen großen Wiedererkennungswert. Es unterstrich mehr als eine Darstellung der eigentlichen Krönungszeremonie, dass Maria Theresia als König von Ungarn ein männliches Amt bekleidete und das Schwert der Gerechtigkeit und der Herrschaft symbolisch wie sprichwörtlich zu führen wusste. Zwar ist der Krönungsritt als Bildmotiv in der Druckgrafik auch bei früheren ungarischen Königen verbreitet, ${ }^{80}$ doch nur Karl III. (VI.) wurde auch auf einer Medaille beim Krönungsritt dargestellt. ${ }^{81}$ Innerhalb der Bildpublizistik zu Maria Theresias Krönung kam diesem Motiv eine überproportionale Bedeutung zu. Da eine Königin mit Schwert auch Maria Theresias Hofbeamten ungewöhnlich erschien, wurde ähnlichen Reaktionen im Adel und in der Bevölkerung durch eine offensive Bildpolitik entgegen gewirkt. Diese Repräsentationsstrategie setzte sich in den folgenden Jahren weiter fort: Maria Theresia legitimierte ihre Herrschaft immer wieder durch die Betonung der dynastischen Kontinuität und ihre erblich bedingte Regierungsbefähigung. Die Herrschaft einer Frau in der Habsburgermonarchie wurde in Bild- und Textmedien als Selbstverständlichkeit kommuniziert.

Seit der Mitte des 18. Jahrhunderts wurde das höfische Festzeremoniell, insbesondere bei Krönungen und Huldigungen, zunehmend kritisch gesehen. Die 
ausgeführten Rituale und Zeremonien erschienen inhaltsleer und rechtlich irrelevant. Gleichzeitig zum Rückgang der rechtsstaatlichen Verbindlichkeit von Krönungszeremonien stieg die repräsentative Pracht diametral an. ${ }^{82}$ Galt dies auch für die ungarische Krönung Maria Theresias?

Angesichts ihrer besonderen Situation als weibliche Thronfolgerin waren die symbolischen Komponenten des ungarischen Krönungszeremoniells politisch aufgeladen. Sie wurden in ihrer traditionellen Überlieferung zur Betonung einer reibungslosen Thronfolge eingesetzt. Die wenigen Anpassungen an ihr weibliches Geschlecht im Zeremoniell betrafen nur Details; in der Außenwahrnehmung durch $\mathrm{Zu}-$ schauer, Botschafter und andere Beobachter wurde ein unverändertes Krönungszeremoniell für einen männlichen König präsentiert, das keinen Spielraum für die Hinterfragung der weiblichen Thronfolge offen ließ. Die wichtigste verfassungsrechtliche Botschaft dieser Inszenierung bestand darin, dass Maria Theresia trotz ihres Geschlechts ein uneingeschränkt herrschender König war. Insofern hatte die Krönung Maria Theresias in der öffentlichen Wahrnehmung eine größere politische Bedeutung als alle bisherigen Königskrönungen in Preßburg. Dass dadurch dem Krönungszeremoniell wieder seine mittelalterliche rechtliche Relevanz zukam, ist jedoch nicht anzunehmen, weil gerade die unproblematische Übertragung eines nur Männern vorbehaltenen Krönungszeremoniells auf eine Frau die Sinnentleerung des Zeremoniells im 18. Jahrhundert unterstreicht. Hingegen zeigt die Krönung Maria Theresias im ersten Regierungsjahr deutlich, dass - anders als bei ihrem Thronfolger Joseph II. - der Krönungsakt als Initiationsritual an sich durchaus Bedeutung besaß, weil ihre ererbten Titel von ausländischen Potentaten als bloße Ansprüche interpretiert und politisch wie militärisch missachtet wurden. So wurde ihr Gegner Karl Albrecht von Bayern noch im selben Jahr zum böhmischen König gekrönt - er war Maria Theresias Ansprüchen durch militärische Erfolge zuvorgekommen. Als rechtsstaatlich anerkannte sowie gekrönte und gesalbte Königin von Ungarn hatte Maria Theresia hingegen nicht nur eine souveräne Königswürde inne, sondern auch eine politische wie militärische Machtbasis für den Österreichischen Erbfolgekrieg gewonnen.

\section{Anmerkungen}

1 Der Artikel entstand im Rahmen des FWF-Projekts P 27512 „Herrscherrepräsentation und Geschichtskultur unter Maria Theresia (1740-1780)“ unter der Leitung von Werner Telesko an der Österreichischen Akademie der Wissenschaften am Institut für kunstund musikhistorische Forschungen, Abteilung Kunstgeschichte.

2 Barbara Stollberg-Rilinger: Verfassung und Fest. Überlegungen zur festlichen Inszenierung vormoderner und moderner Verfassungen, in: Hans-Jürgen Becker (Hg.): Interdependenzen zwischen Verfassung und Kultur (= Beihefte zu „Der Staat“. Zeitschrift für Staatslehre und Verfassungsgeschichte, deutsches und europäisches öffentliches Recht 15), Berlin: Duncker \& Humblot 2003, S. 7-37, hier S. 22-24.

3 Harriet Rudolph: Die Herrschererhebung als Fest. Krönungsfeste im Vergleich, in: Michael Maurer (Hg.): Festkulturen im Vergleich. Inszenierung des Religiösen und Politischen, Köln/Weimar/Wien: Böhlau 2010, S. 13-42, hier S. 20. Bernd Sösemann: Zeremoniell und Inszenierung. Öffentlichkeit und dynastisch-höfische Selbstdarstellung in der preußischen Krönung und den Jubiläumsfeiern (1701-1851), in: Ders. (Hg.): Kommunikation und Medien in Preußen vom 16. zum 19. Jahrhundert (= Beiträge zur Kommunikationsgeschichte 12), Stuttgart: Franz Steiner Verlag 2002, S. 85-135.

4 Horst Carl: Art. „Repräsentation. Allgemein“, in: Friedrich Jäger (Hg.): Enzyklopädie der Neuzeit, Band 11, Stuttgart: Metzler 2010, Sp. 62-64.

5 Barbara Stollberg-Rilinger: Herstellung und Darstellung politischer Einheit. Instrumentelle und symbolische Dimensionen politischer Repräsentation im 18. Jahrhundert, in: Jan Andres/Alexa Geisthövel/Matthias Schwengelbeck (Hg.): Die Sinnlichkeit der Macht. Herrschaft und Repräsentation seit der Frühen Neuzeit (= Historische Politikforschung 5), Frankfurt am Main/New York: Campus 2005, S. 73-92, hier S. 77.

6 Andreas Gestrich: Absolutismus und Öffentlichkeit. Politische Kommunikation in Deutschland zu Beginn des 18. Jahrhunderts, Göttingen: Vandenhoeck \& Ruprecht 1994, S. 118.

7 Gerrit Herlyn: Ritual und Übergangsritual in komplexen Gesellschaften. Sinn- und Bedeutungszuschreibungen zu Begriff und Theorie, Münster: Lit 2002.

8 Ernst Kantorowicz: Die zwei Körper des Königs. Eine Studie zur politischen Theorie des Mittelalters (Deutsche Erstausgabe), München: Dt. Taschenbuch Verlag 1990. Zur Rezeptionsgeschichte vgl. Kristin Marek: Die Körper des Königs. Effigies, Bildpolitik und Heiligkeit, München: Wilhelm Fink 2009, S. 99-113. 
9 Stollberg-Rilinger: Verfassung und Fest (wie Anm. 2), S. 13.

10 Rachel Weil: Der königliche Leib, sein Geschlecht und die Konstruktion der Monarchie, in: Regina Schulte (Hg.): Der Körper der Königin. Geschlecht und Herrschaft in der höfischen Welt seit 1500, Frankfurt am Main/New York: Campus 2002, S. 99-111, hier S. 104.

11 Vgl. Johann Rautenstrauch: Biographie Marien Theresiens, Wien: ohne Verlag 1779, S. 350-360.

12 Pierre Béhar: Die Anfänge des modernen Österreich, in: Ders./Marie-Thérèse Mourey/Herbert Schneider (Hg.): Maria Theresias Kulturwelt. Geschichte, Religiosität, Literatur, Oper, Ballettkultur, Architektur, Malerei, Kunsttischlerei, Porzellan und Zuckerbäckerei im Zeitalter Maria Theresias (= Documenta Austriaca. Literatur und Kultur in den Ländern der ehemaligen Donaumonarchie 2), Hildesheim/Zürich/ New York: Georg Olms Verlag 2011, S. 9-21, hier S. 14. Zur Pragmatischen Sanktion vgl.: Victor-Lucien Tapié: L'Europe de Marie-Thérèse. Du baroque aux lumières, Paris: Fayard 1973, S. 31-42.

13 István Kálly: Die staatsrechtliche Stellung Ungarns und die Landtage unter Maria Theresia, in: Gerda Mraz/Gerald Schlag (Hg.): Maria Theresia als Königin von Ungarn (Ausst.-Kat. Schloß Halbturn), Eisenstadt: Amt der Burgenländischen Landesregierung, Abt. XII/1 - Allgemeine Kulturangelegenheiten 1980, S. 22-29, hier S. 22.

14 Vgl. Alfred von Arneth: Maria Theresia's erste Regierungsjahre, Erster Band 1740-1741, Wien: Wilhelm Braumüller 1863, S. 88 f. Wienerisches Diarium, 22. Oktober 1740, S. 6.

15 Walter Koschatzky: Maria Theresia und ihre Zeit. Zur 200. Wiederkehr des Todestages (Ausst.-Kat. Schloss Schönbrunn), Salzburg/Wien: Residenz-Verlag 1980, S. 60 .

16 Vgl. die Berichte des preußischen Gesandten Podewils an Friedrich II. vom 18. Januar 1747. Carl Hinrichs (Hg.): Friedrich der Große und Maria Theresia. Diplomatische Berichte von Otto Christoph Graf v. Podewils, Königl. Preuss. Gesandter am österreichischen Hofe in Wien, Berlin: R. v. Decker's Verlag, G. Schenk 1937, S. 40.

17 Vgl. Karl Vocelka: Österreichische Geschichte 16991815. Glanz und Untergang der höfischen Welt. Repräsentation, Reform und Reaktion im habsburgischen Vielvölkerstaat, Wien: Ueberreuter 2001, S. 164-170.

18 Vgl. Huldigungen anlässlich von Josephs Geburt durch Illuminationen in der Stadt Wien: Wiennerische Beleuchtungen, oder Beschreibung aller deren Triumph- und Ehren-Gerüsten, Sinn-Bildern und anderen sowol herzlich- als kostbar und annoch nie so prächtig gesehenen Auszierungen Welche bey denen zu Ehren der höchst-gewünschten Geburt Josephi den 13. Martii das erstemal und sodann bey allerhöchst-Ihro Majestät der Königin von Hungarn und Böheim Erz-Herzogin zu Oesterreich und vermählten Herzogin zu Lothringen und Bar Groß Herzogin von Toscana Mariae Theresiae Unseren Allergnädigsten Frauen Frauen beseegneten Hervorgang, Wien: Johann Peter van Ghelen 1741. P.L.C.V.H.: Die durch die neu= aufgegangene Sonne Triumphierende Tugend und Liebe wurde, als die Allerdurchlauchtigste und Großmächtigste Fürstin und Frau, Frau, Maria Theresia [etc.] mit einem so lange gewünschten $\mathrm{Erb}=$ und Cron= Prinzen $[. .$.$] zum Heil und allgemeiner Freude$ dero allergetreuesten Unterthanen beglückseeligte und erfreute, Darnach aber dero hohen königlichen Vorgang hielt, Von einem wohl-edel-hochweisen Stadt-Rath der königl. Haupt- und Residenz-Stadt Wien in einer Illumination vorgestellet und in folgenden Zeilen betrachtet und vorgebildet, Wien: Johann Peter van Ghelen 1741.

19 Österreichisches Staatsarchiv, Haus-, Hof- und Staatsarchiv (im Folgenden: HHStA), Zeremonialprotokolle 18, Fol. $150^{\mathrm{v}}-152^{\mathrm{v}}$.

20 Zu Hofkonferenzen vgl. Andreas Pečar: Die Ökonomie der Ehre. Höfischer Adel am Kaiserhof Karls VI., Darmstadt: Wissenschaftliche Buchgesellschaft 2003, S. 200-207.

21 HHStA, Zeremonialprotokolle 18, Fol. 180 .

22 Štefan Holčík: Krönungsfeierlichkeiten in Preßburg/ Bratislava 1563-1830, Bratislava: Ikar 2005, S. 12. Als Quelle vgl. Friedrich Edelmayer u.a. (Hg.): Die Krönungen Maximilians II. zum König von Böhmen, Römischen König und König von Ungarn (1562/63) nach der Beschreibung des Hans Habersack, ediert nach CVP 7890 (= Fontes Rerum Autriacarum. Österreichische Geschichtsquellen. Erste Abteilung Scriptores 13), Wien: Verlag der Österreichischen Akademie der Wissenschaften 1990, S. 178-205.

23 Rudolph: Herrschererhebung (wie Anm. 3), S. 39-41. Stollberg-Rilinger: Verfassung und Fest (wie Anm. 2), S. 22-24.

24 HHStA, Ältere Zeremonialakten 40, Konv. 12, Hofprotokoll-Extrakt vom 20. und 21. Juni 1741, Fol. 13.

25 Andrea Sommer-Mathis: Residenz und Raum. Höfisches Fest in Wien im Wandel vom 16. zum 17. Jahrhundert, in: Herbert Karner (Hg.): Die Wiener Hofburg 1521-1705. Baugeschichte, Funktion und Etablierung als Kaiserresidenz (= Veröffentlichungen zur Bau- und Funktionsgeschichte der Hofburg II), Wien: Verlag der Österreichischen Akademie der Wissenschaften 2014, S. 494-508, hier S. 496.

26 Harriet Rudolph: Art. „Entrée [festlich, triumphale]“, in: Werner Paravicini (Hg.): Höfe und Residenzen 
im spätmittelalterlichen Reich, Bilder und Begriffe, 1. Teilband: Begriffe (= Residenzenforschung 15.II), Ostfildern: Jan Thorbecke Verlag 2005, S. 318-323. Stollberg-Rilinger: Verfassung und Fest (wie Anm. 2), S. 23.

27 Andrea Sommer-Mathis: „... ma il Papa rispose, che il Re de ${ }^{`}$ Romani a Roma era lui“ Frühneuzeitliche Krönungsfestlichkeiten am Kaiser- und am Papsthof, in: Richard Bösel/Grete Klingenstein/Alexander Koller (Hg.): Kaiserhof - Papsthof (16.-18. Jahrhundert) (unter Mitarbeit von Elisabeth Garms-Cornides, Jan Paul Niederkorn und Andrea Sommer-Mathis), Wien: Verlag der Österreichischen Akademie der Wissenschaften 2009, S. 251-284, hier S. 252.

28 HHStA, Zeremonialprotokolle 18, Fol. $276^{v}$.

29 Ebd., Fol. 292 $-292^{v}$.

30 Stollberg-Rilinger: Verfassung und Fest (wie Anm. 2), S. 14.

31 Veronika Pokorny: Clementia austriaca. Studien zur Bedeutung der clementia princeps für die Habsburger im 16. und 17. Jahrhundert, in: Mitteilungen des Instituts für Österreichische Geschichtsforschung, Nr. 86 (1978), S. 310-364.

32 Rudolph: Herrschererhebung (wie Anm. 3), S. 36 f.

33 Štefan Holčík/Jana Luková/Zuzana Francová: Coronation Festivities/Krönungsfeierlichkeiten. Bratislava/ Preßburg 1563-1830, Bratislava: Múzeum mesta Bratislavy 2015, S. 19.

34 Vgl. HHStA, Ältere Zeremonialakten 40, Konv. 11, Fol. $171^{\mathrm{r}}-180^{\mathrm{v}}$.

35 Ebd., Fol. 172

36 Vgl. Sandra Hertel: Maria Elisabeth. Österreichische Erzherzogin und Statthalterin in Brüssel 1725-1741 (= Schriftenreihe der Österreichischen Gesellschaft zur Erforschung des 18. Jahrhunderts 16), Wien/Köln/ Weimar: Böhlau 2014, S. $301 \mathrm{f}$.

37 HHStA, Zeremonialprotokolle 18, Fol. $305^{\mathrm{v}}$.

38 Ebd., Fol. 170.

39 Holčík: Krönungsfeierlichkeiten (wie Anm. 22), S. 22.

40 HHStA, Zeremonialprotokolle 18, Fol. 180v

41 Ebd.

42 Anette Kuhn: Das Schwert in Frauenhand, in: Gabriele Frohnhaus/Barbara Grotkamp-Schepers/Renate Philipp (Hg.): Schwert in Frauenhand. Weibliche Bewaffnung (= Dokumentation des Symposiums am 4.12.1998 im Deutschen Klingenmuseum Solingen), Solingen: Deutsches Klingenmuseum 1999, S. 7-14, hier S. 7.

43 Barbara Degen: Justitia ist eine Frau. Geschichte und Symbolik der Gerechtigkeit (= Katalog zur Ausstellung „Füllhorn, Waage, Schwert - Justitia ist eine Frau“ im Haus der Frauengeschichte), Opladen: Verlag Barbara Budrich 2008, S. 114.
44 Vgl. Gestrich: Absolutismus und Öffentlichkeit (wie Anm. 6), S. 125.

45 HHStA, Zeremonialprotokolle 18, Fol. 285"

46 Ebd., Fol. 287 .

47 Rudolph: Herrschererhebung (wie Anm. 3), S. 29.

48 Gestrich: Absolutismus und Öffentlichkeit (wie Anm. 6), S. 118.

49 Stollberg-Rilinger: Verfassung und Fest (wie Anm. 2), S. 24.

50 Holčík: Krönungsfeierlichkeiten (wie Anm. 22), S. 36.

51 HHStA, Zeremonialprotokolle 18, Fol. 299".

52 Frank Bösch: Europäische Medienereignisse, in: Europäische Geschichte Online (EGO), hg. vom Institut für Europäische Geschichte (IEG), Mainz 2010-12-03. URL: http://www.ieg-ego.eu/boeschf-2010-de [201604-18]. Thomas Weißbrich/Horst Carl: Präsenz und Information. Frühneuzeitliche Konzeptionen von Medienereignissen, in: Joachim Eibach (Hg.): Europäische Wahrnehmungen 1650-1850. Interkulturelle Kommunikation und Medienereignisse, Hannover: Wehrhahn 2008, S. 75-98.

53 Volker Bauer: Höfische Gesellschaft und höfische Öffentlichkeit im alten Reich. Überlegungen zur Mediengeschichte des Fürstenhofs im 17. und 18. Jahrhundert, in: Jahrbuch für Kommunikationsgeschichte 5 (2003), S. 29-68. Volker Bauer: Nachrichtenmedien und höfische Gesellschaft. Zum Verhältnis von Mediensystem und höfischer Öffentlichkeit im Alten Reich, in: Johannes Arndt/Esther-Beate Körber (Hg.): Das Mediensystem im Alten Reich der Frühen Neuzeit (1600-1750) (= Veröffentlichungen des Instituts für europäische Geschichte Mainz, Abteilung für Universalgeschichte, Beiheft 75), Göttingen: Vandenhoeck \& Ruprecht 2010, S. 173-194, hier S. 174.

54 Sebastian Küster: Vier Monarchien - Vier Öffentlichkeiten. Kommunikation um die Schlacht bei Dettingen, Münster: Lit 2004, S. 473.

55 Holger Böning: Der „gemeine Mann“ als Zeitungsund Medienkonsument im Barockzeitalter, in: Arndt/ Körber: Mediensystem (wie Anm. 53), S. 227-238, hier S. $227 \mathrm{f}$.

56 Ernst Opgenoorth: Publicum - privatum - arcanum. Ein Versuch zur Begrifflichkeit frühneuzeitlicher Kommunikationsgeschichte, in: Sösemann: Kommunikation und Medien (wie Anm. 3), S. 22-44, hier S. 42.

57 Sösemann: Zeremoniell und Inszenierung (wie Anm. 3), S. 96.

58 Stefan Seitschek: „Einige caeremonialpuncten bet(reffend)“. Kommunizierende Gefäße: Zeremonialprotokoll und Wiener Diarium als Quelle für den Wiener Hof (18. Jh.), Wien: Dipl.-Arb. 2011, S. 56-58.

59 Jürgen Wilke: Die Zeitung, in: Ernst Fischer/Wilhelm Haefs/York-Gothart Mix (Hg.): Von Almanach bis 
Zeitung. Ein Handbuch der Medien in Deutschland 1700-1800, München:Beck1999,S.388-402, hierS.398.

60 Stefan Seitschek: Religiöse Praxis am Wiener Hof. Das Beispiel der medialen Berichterstattung, in: István Fazekas u.a. (Hg.): Frühneuzeitforschung in der Habsburgermonarchie. Adel und Wiener Hof - Konfessionalisierung - Siebenbürgen (= Publikationen der Ungarischen Geschichtsforschung in Wien VII), Wien: Institut für Ungarische Geschichtsforschung in Wien 2013, S. 71-101, hier S. 75.

61 Ebd., S. 125. Bauer: Nachrichtenmedien (wie Anm. 52), S. 179.

62 O. A.: Ausführliche Beschreibung der den 25. Junii in der königlichen ungarischen freyen Reichs=Stadt Preßburg glücklich vollzogenen prächtigen Crönung Ihrer Allerdurchleuchtigsten Majestät Maria Theresia Ertz=Hertzogin zu Oesterreich, vermählten Hertzogin $\mathrm{zu}$ Lothringen und Baar Groß=Hertzogin zu Toscana zur Königin in Hungarn, Wien: Johann Peter van Ghelen 1741.

63 Wienerisches Diarium, 15. Juli 1741, S. 10.

64 Beispielsweise erschien 1741 eine Version der Krönungsbeschreibung in der Freien Reichsstadt Augsburg, gedruckt bey Bernhardus Homodeus Mayer, Buch- und Zeitungsdrucker des Reichsvikariats.

65 Ausführliche Beschreibung (wie Anm. 62), BlattNr. 611-612.

66 HHStA, Zeremonialprotokolle 18, Fol. $308^{\mathrm{r}-\mathrm{v}}$.

67 Sösemann: Zeremoniell und Inszenierung (wie Anm. 3), S. 106. Gestrich: Absolutismus und Öffentlichkeit (wie Anm. 6), S. 125.

68 Christine Vogel: „Evenemens mémorables“ Mediale (Selbst-)Inszenierungen des Parlement de Paris in der Auseinandersetzung mit Ludwig XV., in: Dies./Herbert Schneider/ Horst Carl (Hg.): Medienereignisse im 18. und 19. Jahrhundert. Beiträge einer interdisziplinären Tagung aus Anlass des 65. Geburtstags von Rolf Reichardt, München: De Gruyter 2009, 19-32, hierS.20.

69 Eduard Holzmair: Die offiziellen österreichischen Krönungs- und Huldigungspfennige seit Kaiser Joseph I., in: Jahrbuch der Kunsthistorischen Sammlungen in Wien 50 (1953), S. 199-210.

70 Eduard Fiala: Katalog der Münzen- und Medaillen-Stempel-Sammlung des K.K. Hauptmünzamtes in Wien, Zweiter Band, Wien: Kaiserlich-Königliche Hof- und Staatsdruckerei 1902, S. 244.

71 Heinz Winter: Glanz des Hauses Habsburg. Die habsburgische Medaille im Münzkabinett des Kunsthistorischen Museums (= Kataloge der Medaillensammlung 1), Wien: Kunsthistorisches Museum 2009, S. 86.
72 Thomas Rahn: Psychologie des Zeremoniells. Affekttheorie und Pragmatik in der Zeremoniellwissenschaft des 18. Jahrhunderts, in: Jörg Jochen Berns/Thomas Rahn (Hg.): Zeremoniell als höfische Ästhetik in Spätmittelalter und Früher Neuzeit (= Frühe Neuzeit 25), Tübingen: Niemeyer 1995, S. 74-98, hier S. 77 f. Vgl. auch Gestrich: Absolutismus und Öffentlichkeit (wie Anm. 6), S. 45.

73 Ausführliche Beschreibung (wie Anm. 62), Blatt-Nr. 614.

74 Franz Peikhardt, Lob- und Dankrede Gott dem allerh. für die glückliche Krönung I. k. Maj. Mar. Theresia zur Ungarischen Königin, Wien: Heyinger 1741.

75 Daniel Stadler: Danck- und Glückwunschs-Rede von wegen zu Preßburg, den 25. Junii 1741. vorgegangener höchst-beglückter Crönung seiner königl. Majestät zu Hungarn und Böheimb [...] Maria Theresiae, Ertz-Hertzogin zu Oesterreich etc. [...] bey dem von löbl. vorder-österr. Regierung angestellten DanckFest, Freyburg im Breyßgau: Franz Xaver Schaal 1741.

76 Relationis Historicae Semestralis Autumnalis Continuatio, Jacobi Franci historische Beschreibung der denckwürdigsten Geschichten, so sich in Hoch und Nieder Teutschland [...] etc. vor und zwischen jüngst verflossenen Oster-Meß 1741 biß an die Franckfurter Herbst-Meß dieses lauffenden 1741 Jahrs [...] zugetragen, Frankfurt am Main: Engelhardische Erben 1741, S. $46 \mathrm{f}$.

77 Wienerisches Diarium, 8. Juli 1741, S. 8 und 15. Juli 1741, S. 10.

78 Ausführliche Beschreibung (wie Anm. 62), Blatt-Nr. 615.

79 Winter: Glanz des Hauses Habsburg (wie Anm. 71), S. 85 f. Fiala: Katalog (wie Anm. 70), S. 244 f. Géza Pálffy: Coronatus Posonii. Bratislavské korunovačné medaily a žetóny, (1563-1830), Bratislava: Vydané Slovenským národným múzeom-Historickým, 2014, S. 129-133.

80 Friedrich Polleroß: Austriacus Hungariae Rex. Zur Darstellung der Habsburger als ungarische Könige in der frühneuzeitlichen Graphik, in: Orsolya Bubryák (Hg.): „Ez világ, mint egy kert ...“: tanulmányok Galavics Géza tiszteletére, Budapest: Gondolat 2010, S. 63-78, hier S. 67. Holčík: Krönungsfeierlichkeiten (wie Anm. 22), S. 21.

81 Pálffy: Coronatus (wie Anm. 79), S. 111.

82 Stollberg-Rilinger: Verfassung und Fest (wie Anm. 2), S. $26 \mathrm{f}$. 